\title{
Nurturing Secondary School Student Computational Thinking Through Educational Robotics
}

\author{
https://doi.org/10.3991/ijet.v17i03.27311 \\ Dayang N A Jawawi ${ }^{1(\bowtie)}$, Nurul N Jamal ${ }^{1}$, Shahliza Abdul Halim ${ }^{1}$, \\ Nor Azizah Sa'adon ${ }^{1}$, Rosbi Mamat ${ }^{2}$, Mohd Adham Isa ${ }^{1}$, Radziah Mohamad $^{1}$, \\ Haza Nuzly Abdull Hamed ${ }^{1}$ \\ ${ }^{1}$ School of Computing, Universiti Teknologi Malaysia, Kuala Lumpur, Malaysia \\ ${ }^{2}$ School of Electrical Engineering, Universiti Teknologi Malaysia, Kuala Lumpur, Malaysia \\ dayangeutm.my
}

\begin{abstract}
Digital literacy is becoming a key factor in today's digital revolution age. Computational Thinking (CT) is a new digital literacy that is gradually being introduced in the school curriculum due to its applicability in the daily problem-solving process. Educational Robotics (ER) has been increasingly used as a pedagogical tool to attract students to learn computer programming, and when integrated with CT, they can be used to develop high order thinking skills. However, intertwining between $\mathrm{CT}$ and ER remains a challenge for teachers. This paper describes a method to expose secondary school children to CT concepts and skills through ER learning activities. The method integrates the four CT core concepts, which are problem analysis and algorithm; abstraction; pattern recognition; and decomposition, in a two days' ER workshop implementation. The result of the study shows that the method of integrating CT with ER has the potential to nurture students' CT and programming skills. This study shows a statistically significant increase in the students' understanding of the two CT concepts which are pattern recognition and decomposition concepts.
\end{abstract}

Keywords - computational thinking, Educational Robotics, teaching computer programming

\section{Introduction}

In recent years, Educational Robotics (ER) has been increasingly used as a pedagogical tool [1] to boost the performance of students in several subjects and age levels. ER can be used to motivate and attract students to learn more [2] and improve their learning efficiency further. Many authors reported successful attempts [1] in introducing ER as a non-conventional way to help students learn subjects specifically for science, technology, engineering, and math (STEM) disciplines. Robotics are becoming a wellknown tool [3] in teaching and learning, for a primary and secondary schools in the areas of science and technology as well as several areas in universities engineering courses. These robots are used as a method of active learning that permits several sub- 
jects such as math, computer science, mechanics, technology, electronics, programming, artificial intelligence, and computer vision among others, to be combined as a single subject with a distinct goal.

In this digital revolution age, Computational Thinking (CT) is introduced as a fundamental skill used by many professions. CT is used as a strategy for solving a problem in computing. Wing [4] introduced CT as an approach to solving problems, design systems, and understand human behavior that draws on the concepts fundamental to computing.

Realizing the importance of CT and the benefits of ER, this work presents a method to introduce and nurture CT skills among secondary school students through ER teaching and learning activities. ER exposes students to CT by involving them in the problem-solving process using CT core concepts which are abstraction, algorithm, automation, decomposition, debugging, and generalization [5]. ER can make CT concepts concrete and become a tool that offers opportunities for school students to engage and develop skills in CT.

This paper studies the perspective of using ER as a tool to support the learning of introductory programming by integrating CT in ER through teaching and learning activities. In addition, this paper also describes the implementation of the integration method (CT in ER) in secondary school workshops' activities. The implementation of ER requires the teaching and learning method to employ (1) a mobile robot, (2) teaching modules, and (3) an assessment instrument in a two-day program that consists of a problem-solving workshop and robotic game activities. The implementation of the method gives focus to a short duration teaching and learning session aiming to nurture and introduce CT skills. There are many research works targeting secondary school students because most students around this age can perform the CT and ER activities successfully [6]. Unfortunately, there is a limited research study on short-duration teaching and learning sessions. The implementation of the method in teaching and learning activities for a short duration session was analysed to validate the student's perspective towards the learning and determined by evaluating the students' understanding of CT core concepts.

\section{Background}

\subsection{Educational robotic evolution}

The idea of robotics integration in education has been proposed more than a decade ago [7]. Robotics was earlier used in competition [8] before being employed in early childhood education as a tool to teach and deliver concepts [9]. It has been used as a non-conventional way of attracting students' interest in science, technology, engineering, and mathematics (STEM) education as it will have a high impact on students' future engagement with technology [1]. In today's fourth industrial revolution era, robotics has been a famous supporting tool in education in giving excitement in learning and teaching. The involvement of robotics in education gave benefits to the industries and market. The market is driven primarily by factors such as the use of robots in the field 
of robotics for educational purposes and technological advances [10]. Besides that, ER also has been used in supporting the delivery of learning CT in recent years [11]. Figure 1 shows the evolution of ER from its early days until its integration to support 4IR evolution.

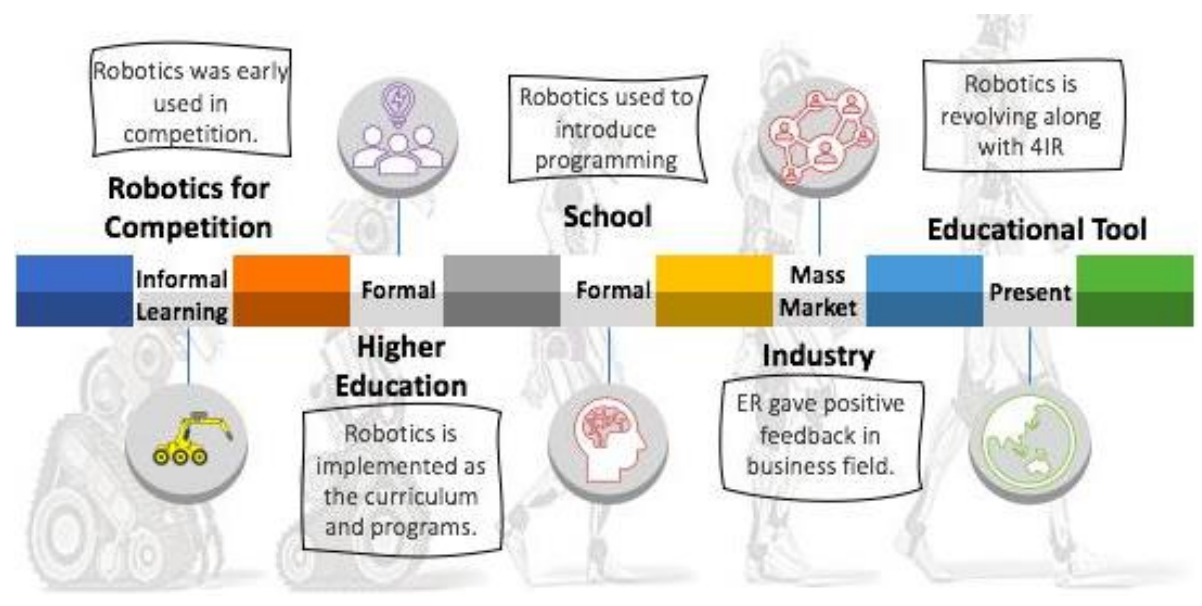

Fig. 1. Evolution of Educational Robotics

Realizing the benefits of robots in teaching, in 2008, Universiti Teknologi Malaysia (UTM) has introduced ER in a formal class education for computing students, and in 2012, ER Co-Curricular Service-Learning program [12] was initiated for teaching problem solving and computer programming for school students. A mobile robot called RoboKar has been used as a tool for educational purposes to promote computer programming to the community including school and college students. RoboKar has evolved from ApiBot mobile robot initially used in a research case [13] into RoboTar in 2008 to be used as a pedagogical tool for undergraduates' formal learning in Embedded and Real-Time programming courses.

\subsection{Computational thinking and educational robotics}

Computing is a ubiquitous technology, and a basic understanding of the technology will help in daily lives. Computational thinking in education is seen as an important skill as today's youths will not only get to work in computer-influenced areas, but they will also need to deal with computers in their daily lives in today's global economy [14]. In school, CT is aimed to help teachers guide the students in searching for new kinds of problems solving techniques for unique or new problems. Many proposed curricular frameworks nurture CT in education. For instance, Duncan et al [15] applied logical reasoning and evaluation concepts of CT in their study. Because CT is a wellestablished technology, many researchers are attempting to study its concepts and attempt to apply them to educational frameworks to enhance youth performance. One of the attempts is to nurture CT through ER to develop higher-order cognitive skills among youth is by Chalmers [16]. 
Different methods are proposed by researchers to study the potential of ER to promote the development of CT skills. Chalmers [16] investigated how Australian primary school teachers taught coding through robotics in the classroom and observed whether the activities gave an impact on the students' CT skills. The finding shows that the use of robots through simple activities helped the teachers to develop student's confidence and improve their knowledge of CT. Another study by Chalmers [16] focused on how teachers can integrate ER and CT. Many other works focused on student development in CT skills through ER at different levels and regions. Noh and Lee [17] conceived a robot programming course for Korean elementary school students and investigated the effectiveness of implementing it and the student's perception towards it in actual classes. Umaschi Ber et al. [18] studied the effectiveness of TanginleK Robotics Program to attract the interest of kindergarten children in learning CT. Most of the findings show that ER courses resulted in positive interest in students' CT learning. Besides focusing on teaching and learning activities, a study conducted by Chen et al. [19] in the United States proposed an instrument to assess elementary students' CT in reasoning and robotics programming. All studies focused on different types of age groups, regions, and education systems which gave it a difficulty to draw a general conclusion for the findings. Therefore, there is a need to study the potential of ER to nurture the development of CT skills in Malaysian secondary school students via short duration workshop activities.

\section{Methodology}

Figure 2 depicts the procedures used to conduct the study which involves data gathering, training sessions, game activities, and evaluation. The main goal of this study is to measure the students' perspective of CT integration in ER during teaching and learning activities and investigate the benefits of ER in learning computer programming. Generally, the study started with data collection that is aimed to have data on students' background and related knowledge. They will undergo a training session on problemsolving using RoboKar and be given time to complete simple programming tasks during robotic game activities. The students will be evaluated using the results of the problem-solving task and post-survey questionnaire. The participants and implementation procedures used in the training and game activities will be described in the next subsections, respectively.

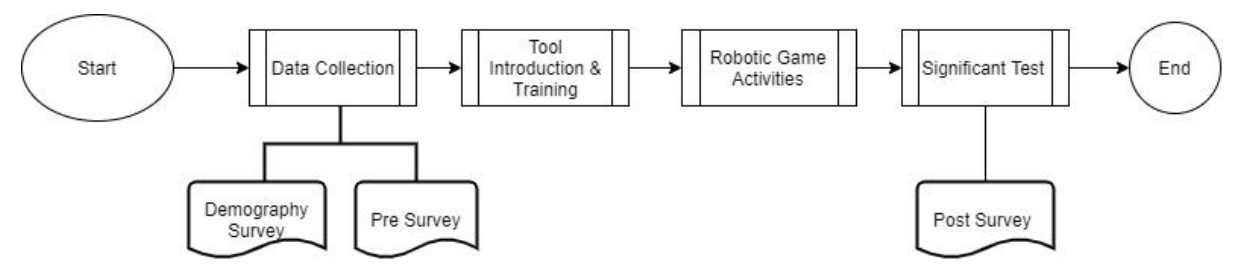

Fig. 2. Data collection and workshop activities 


\subsection{Participants}

A two-day robotic programming training workshop for secondary schools in Segamat, Johor, Malaysia was conducted for the purpose of this study. In total, 75 students from five different secondary schools participated in the workshop. The $75 \mathrm{stu}-$ dents were divided into two categories of groups, Group A and Group B which have the background knowledge in learning $\mathrm{CT}$ before the workshop (N-31) and have no experienced and knowledge in learning $\mathrm{CT}$ (N-44) respectively. Two different backgrounds of groups were selected in purpose to evaluate the effectiveness of the module delivery in learning CT through ER based on the perception of the students. The result of the workshop is a piece of good evidence to prove students' understanding of CT after and before the workshops. Table 1 shows the demographic data of the students.

Table 1. Demographic data of the students

\begin{tabular}{|c|c|c|c|c|c|}
\hline \multicolumn{5}{|c|}{ Demography Data } \\
\hline \multirow{2}{*}{ Question } & \multicolumn{2}{|c|}{ Group A ( Have learned CT) } & \multicolumn{2}{c|}{ Group B ( Have no learned CT) } \\
\cline { 3 - 6 } \multicolumn{2}{|c|}{} & Frequency (N-31) & \% Frequency Distribution & Frequency (N-44) & \% Frequency Distribution \\
\hline \multirow{2}{*}{ Age } & $13-15$ & 26 & 83.71 & 3 & 6.82 \\
\cline { 2 - 6 } & $16-18$ & 5 & 16.29 & 41 & 93.18 \\
\hline
\end{tabular}

\subsection{Implementation procedure}

RoboKar is a small mobile robot developed by Universiti Teknologi Malaysia (UTM) to teach problem-solving through computer programming. As shown in Figure 3 , RoboKar is a tool that has been used in many outreaches' demonstration and activities on programming and CT. This mobile robot is equipped with optical sensors to detect black tracks on the floor and provide direction information for the robot to navigate a track. RoboKar is powered by two small motors which are connected to the right and left wheels. A microcomputer, which is the brain of RoboKar is a small microcomputer Arduino Uno. Arduino microcomputer used on RoboKar can be programmed using the Arduino IDE and the programs written using this IDE can then be uploaded into the Arduino's permanent memory via a USB cable. The c programming language supported by Arduino IDE is used to program a RoboKar.

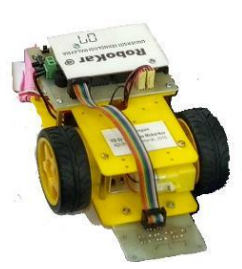

Fig. 3. RoboKar mobile robot

Five RoboKar teaching modules were implemented during the workshop to deliver CT concepts to the workshop's participants. The modules include an introduction to 
RoboKar; algorithm and problem solving; basic C programming; and RoboKar programming and debugging. An active learning approach was employed where the facilitator actively engages students to perform the listed modules. Six activities were designed for students to apply modules' content to solve RoboKar problems. The RoboKar problem-solving activities involve algorithm design, programming, and debugging. Besides the workshop, a game competition that involved RoboKar was held. The gamification of learning approach was implemented to achieve students' learning objectives. A track or line following mobile robot game was designed for a learning activity. To win the game, students should prepare a $\mathrm{C}$ computer program to ensure RoboKar follows the track and pass through all checkpoints and obstacles. Figure 4 shows the track example used in RoboKar's problem-solving activity and game activity. Various simple and complex tracks were made for this workshop.
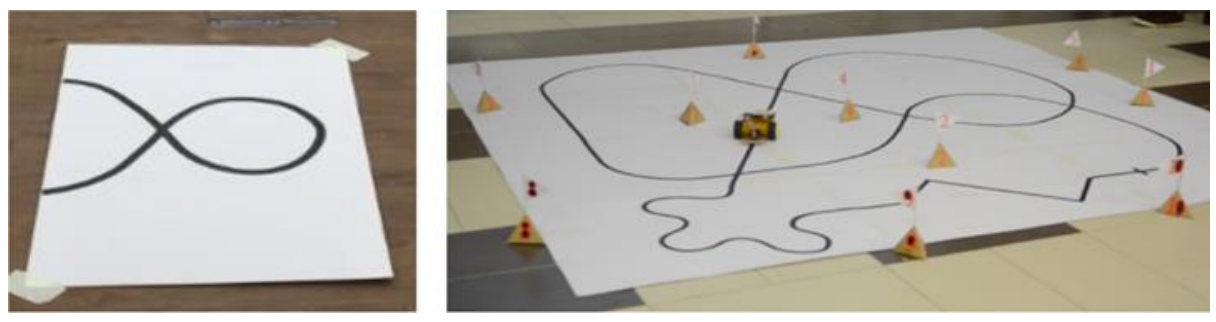

Fig. 4. Example of tracks

Then, Figures 5 (a) and (b) show the actual problem-solving workshop and robotic game activities conducted. At the end of the activities, students were able to achieve the learning objectives which are to (1) solve a simple problem with CT concepts in a given time frame using $\mathrm{C}$ programming language and software development tools; and (2) construct and develop a simple program based on $\mathrm{C}$ programming language which involved variable, simple selection, loop and function concepts. These activities promote the elements aligned by CT. During the workshop, students are nurtured with CT skills through RoboKar. This workshop instills four CT concepts during the students' RoboKar problem-solving process, the CT concepts are problem analysis and algorithm; abstraction, pattern recognition, and decomposition.

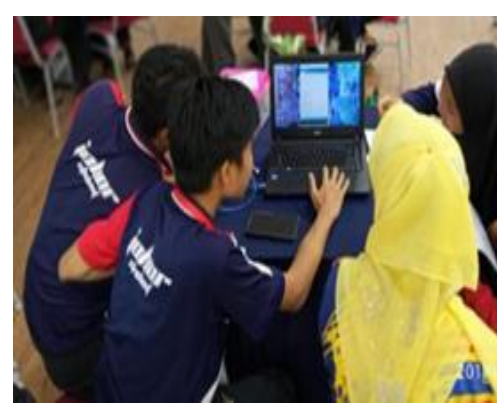

(a)

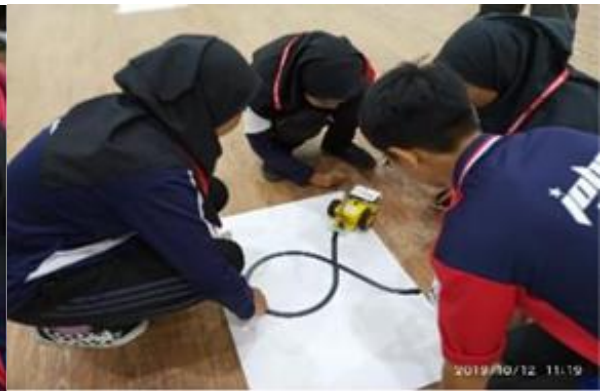

(b)

Fig. 5. Robotic programming activities 


\subsection{A model for CT}

Besides, there are few examples of curricular framework that suggests CT basic concepts or skills in education. An example of the framework is the CT framework by College Board created for U.S. high school course on Computer Science Principles course in 2010. Followings are the description of the few core concepts of CT by Joke et al [20]:

- Decomposition is the method of breaking down larger tasks into smaller and easier tasks so that they can be understood and solved.

- Pattern recognition is the ability to recognize and see the similarities and regularities of the subject, for example in data.

- Abstraction can be defined as the ability to filter unnecessary information and give more focus to relevant information that defines the key ideas.

- Algorithm concept is the ability to develop one set of a procedure or step-by-step procedure that needs to be taken to solve a problem.

In this study, we focused on four CT core skills which are (1) problem analysis and algorithm, (2) abstraction, (3) pattern recognition, and (4) decomposition. Aside from that, we introduced the four skills in the context of ER learning activities that have been implemented during robotic programming workshops and game activities. Intertwining $\mathrm{CT}$ and ER can be led by the development of the four skills. The problem analysis and algorithm skill will help them in constructing instruction on how to do something step by step to solve a problem. The students were given the task of instructing RoboKar to detect and move on a black line track using $\mathrm{C}$ programming. They were taught to do a simple flowchart to see the pre-presentation of the robot's movement. Students had to analyze the behavior of RoboKar and all possible problems, such as the pattern of track and time taken for RoboKar to complete the mission.

In programming activities, high-level abstraction modules abstracted the complexity of the RoboKar programming process by removing unnecessary detail to identify the solution. We introduced the use of programming function and procedure to support the abstraction concept in their problem-solving process by hiding the full complexity of the problem. A set of pre-defined functions were introduced in their problem-solving process to allow knowledge on robot hardware to be abstracted and ensure that students can write programs easily and focus on solving the problem at hand.

The algorithm and programming solution developed by the students in the workshop is aimed to be used for multiple track pattern as the different track has a different challenge. Thus, students need to identify the patterns and commonalities of the solutions first, to see the adaptation of the solution into one problem or another problem. Decomposition is defined as a process by which a larger task is broken down into smaller and simpler tasks so that it can be understood and solved. A classical decomposition technique, Sense, Plan, Act [21], was introduced to enable students to decompose the complex RoboKar problem into a set of simple robot behaviors and implement the simple behaviors using $\mathrm{C}$ programming. 


\subsection{Data collection and analysis}

This study collected students' perceptions and understanding of solving problems based on CT concepts using RoboKar. A set of pre-and post-survey questionnaires have been developed. The questionnaires consist of a Likert scale and open-ended questions. The survey is based on a 4-point Likert scale that ranges from Totally Agree-4, Agree3 , Disagree-2, and Totally Disagree- 1 . The main question is on the implementation of the CT concepts when solving the programming problem. Using the statement "When solving the problem, I...", the questions were divided into four elements which focus on CT concepts, namely: (i) Construct step-by-step algorithm for problem-solving, (ii) Abstract the unnecessary details in problem-solving, (iii) Recognize the pattern of the problem (specifically the track pattern), and (iv) Decompose the problem. Meanwhile, the open-ended questions were applied to identify students' feedback on using robotic methods in solving a problem.

A paired-samples t-test was conducted to investigate the differences in the understanding of the students before and after attending this two-day workshop. In measuring the significant value $(>0.05$ or $<0.05)$ of the data collected based on the Likert scale survey, a null hypothesis, and alternative hypothesis were designed which are " $\mathrm{HO}$ : There is no difference in the students understanding before and after the workshop", and "H1: There is the difference on the students understanding before and after the workshop". Besides that, this study also implemented Cronbach's alpha technique to measure the reliability level recommended by Peterson [22] as shown in Table 2.

Table 2. Reliability Level of Cronbach's alpha

\begin{tabular}{|l|c|}
\hline Reliability Level & Internal Consistency Acceptance \\
\hline Below 0.6 & Unacceptance level \\
\hline $0.6-0.7$ & Low acceptance level \\
\hline $0.8-0.9$ & Moderate to High Acceptance \\
\hline Above 0.9 & High Acceptance Level \\
\hline
\end{tabular}

\section{$4 \quad$ Result and discussion}

As mentioned, paired sample t-test was used to measure the significant value for each CT concept obtained by the two groups. The analysis showed that there is no change for Group A. Students from Group A have learned CT before attending the workshop. All significant values for the four concepts are greater than 0.05 , thus the null hypothesis needs to be retained whereas there is no significant difference in the students' understanding of both concepts before and after the workshop based on Jovancic's guideline. Meanwhile, Group B shows a statistically significant increase in pattern recognition and decomposition concepts which resulted in a p-value smaller than 0.05 which are 0.048 and 0.009 respectively. Thus, the null hypothesis needs to be rejected. It has been proven that the workshop helps the students understand the two CT concepts. For the other two concepts which are algorithmic thinking and abstraction, there is no difference in the understanding of the students from Group B. 
However, from the graph analysis in Figure 6, it is observed that $4.54 \%$ of the students responded 'disagree' with the statement - "When solving the problem, I construct step by step for problem-solving”. Meanwhile, the given post-survey questionnaire shows a decrease in the percentage of students who disagree with the statements to $2.27 \%$. This shows a slight improvement in the student understanding but not statistically significant. Next, the observation on abstraction concept understanding in Figure 6 , shows an increase in the students' negative perception from $2.27 \%$ to $4.54 \%$. This indicated the students have difficulty in applying this concept in RoboKar activities. This is due to the difficulty to introduce the abstraction concept in this short period of time, were in this session, they only used the existing functions for hardware abstract and do not implement their own functions in problem-solving activities. The abstraction $\mathrm{CT}$ concept requires more time to understand and implement due to the technical complexity in $\mathrm{C}$ programing language function definition, declaration, and parameter passing.

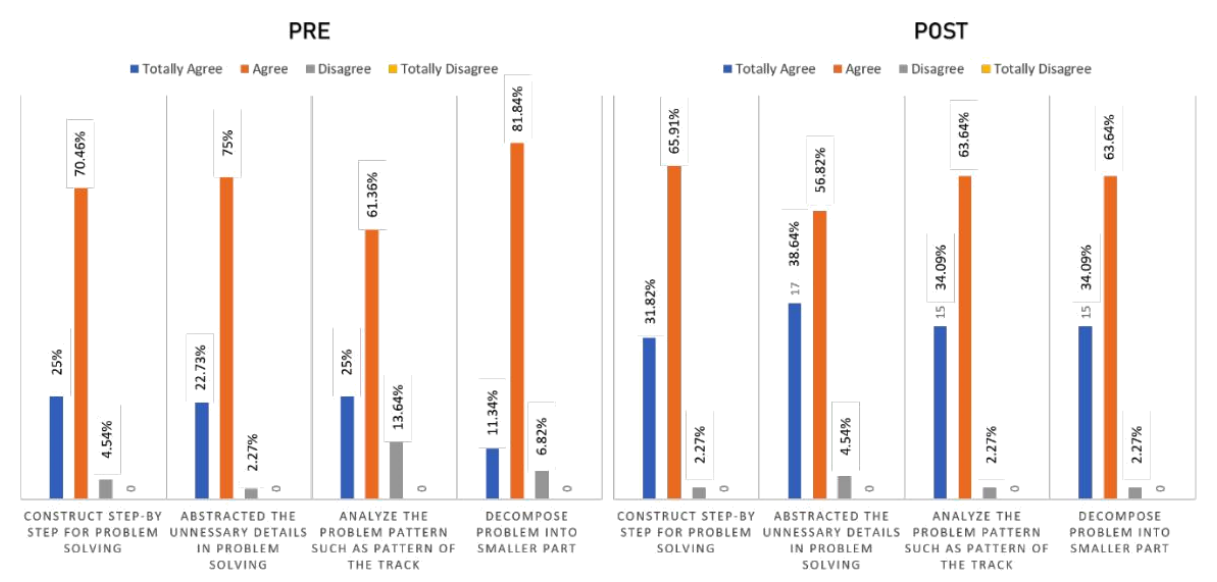

Fig. 6. Pre- and post-survey results for Group B (N-44)

In this study, the reliability assessment was done using Microsoft Excel. Group A and Group B obtained a high acceptance level of reliability, 0.953 and 0.902 respectively, showing that the survey is considered reliable. Furthermore, based on the survey, $99 \%$ of the students gave positive feedback that robotics programming activities are able to help them to solve problems. Even though, most of the students $(60 \%)$ who participated in the workshop are not familiar with CT concepts before, but almost all agreed these CT concepts do help them to solve problems. Via this survey, it is proven that CT can be taught to students by applying the robotics problem task. For instance, students were able to solve robotics problems via the application of basic CT concepts such as step-by-step processes and decompose problems into parts.

Figure 6 illustrates an improvement based on the difference percentage of the students that agree before the workshop and after the workshop. The open-ended questions feedbacks concluded that the students could identify the four core CT concepts in their problem-solving activities. This feedback result matches with the students' perception 
result in Figure 6. The result of the study is able to show that, nurturing CT through ER is a good opportunity for the students to improve themselves with good skills and prepare them in facing a real-problem situation. Thus, the learning can be adopted in the future as an initiative to upgrade their skills.

Due to the pandemic situation, there are challenges for delivering the knowledge to the students as the current learning environment has been shifted to online. Thus, another consideration that needs to be taken in the future is to develop an online platform that supports the learning CT through ER anywhere, anytime, and any place for the students. The new norm process in learning gave the challenge in delivering the knowledge to the students. Besides that, it is also a challenge for the researchers in integrating the ER as a physical tool into the online platform. Thus, an ubiquitous learning environment can be considered to be implemented to cater the situation.

\section{Conclusion}

In this study, the integration of CT core concepts into ER was described. Implementation of the proposed method in teaching and learning activities was analyzed using pre-and post-questionnaires done for a two days' workshop. The aim of the workshop is to improve students' perception and understanding of solving the problem based on CT concepts using RoboKar. The result shows a positive and significant impact on students' CT skills in pattern recognition and decomposition concepts for the group of students with no prior knowledge of CT. For the other two CT concepts which are algorithmic thinking and abstraction, it is difficult to introduce the concept in a short period of teaching and learning sessions using ER. The analysis has explored the integrated ER with digital CT learning activities and an active learning method to engage students in problem-solving while introducing and nurturing CT skills among school students. In future works, the authors wish to adapt the method by taking more activities into consideration and use more precise experiments designed to control possible factors that might affect students' learning performance.

\section{Acknowledgment}

We fully acknowledge Universiti Teknologi Malaysia UTMHR Grant Vot No. 08G67 and Office of ADUN Kemelah, Segamat, Johor for the financial support, which has made this research endeavor possible. We thank the school students and teachers who participated in the workshop, UTM's students who served as facilitators, and members of the RoboKar team and Software Engineering Research Group (SERG) for their help. 


\section{$7 \quad$ Reference}

[1] Jamal N. N, Jawawi D. N., Hassan R. \& Mamat, R. (2021). "Conceptual Model of Learning Computational Thinking through Educational Robotic.” Int. Journal of Emerging Technologies in Learning, vol. 16, pp. 91-106. https://doi.org/10.3991/ijet.v16i15.24257

[2] Chin, K., Hong, Z., \& Chen, Y. (2014). "Impact of using an educational robot-based learning system on students' motivation in elementary education." Learning Technologies. https://doi.org/10.1109/TLT.2014.2346756

[3] Sell R., Seiler S. \& Ptasik D. (2012). "Embedded System and Robotic Education in a Blended Learning Environment Utilizing R emote and Virtual Labs in the Cloud, Accompanied by 'Robotic HomeLab Kit'.' Int. Journal of Emerging Technologies in Learning, vol. 7, pp. 26-33. https://doi.org/10.3991/ijet.v7i4.2245

[4] Wing, J. M. (2006). “Computational Thinking." Communications of the ACM, 33-35. https://doi.org/10.1145/1118178.1118215

[5] Bocconi, S., Chioccariello, A., Dettori, G., Ferrari, A., \& Engelhardt, K. (2016). "Developing Computational Thinking in Compulsory Education. ”. Spain: European Unian.

[6] Atmatzidou, S., \& Demetriadis, S. (2016). "Advancing students' computational thinking skills through educational robotics: A study on age and gende relevant differences." Robotics and Autonomous Systems, 661-670. https://doi.org/10.1016/j.robot.2015.10.008

[7] Miglino, O., Lund, H., \& Cardaci, M. (1999). "Robotics as an Educational Tool." Association for the Advancement of Computing in Education.

[8] Yudin, A., Vlasov, A., Salmina, M., \& Shalashova, M. (2020). "Evolution of Educational Robotics in Supplementary Education of Children." Robotics in Education, 336-343. https://doi.org/10.1007/978-3-030-26945-6_30

[9] Papadakis S. (2020). "Robots and Robotics Kits for early Chidhood and First School Age" Int. Journal of Interactive Mobile Technologies, vol. 14, pp. 34-56. https://doi.org/10.3991 lijim.v14i18.16631

[10] MarketsandMarkets Research Private Ltd. (2018, March). Educational Robot Market. Retrieved from Markets Research Firm: https://www.marketsandmarkets.com/Market-Reports/educational-robot-market-28174634.html

[11] Grandi, R., Falconi, R., \& Melchiorri, C. (2014). "Robotic Competitions: Teaching Robotics and Real-Time Programming with LEGO Mindstorms." Proceedings of 19th World Congress, The International Federation of Automatic Control Cape Town, South Africa. South Africa. https://doi.org/10.3182/20140824-6-ZA-1003.00222

[12] Jawawi, D. N., Mamat, R., Ridzuan, F., Khatibsyarbini, M., \& Zaki, M. (2015). "Introducing computer programming to secondary school students using mobile robots." Control Conference (ASCC), 2015 10th Asian (pp. 1-6). IEEE. https://doi.org/10.1109/ASCC.2015. $\underline{7244750}$

[13] Jawawi, D. N. A. Mamat, R., (2009), "Teaching Embedded Real-Time Programming using Autonomous Mobile Robots for Undergraduates in Computer Science.” Proceedings International Conference on Control, Instrumentation and Mechatronics Engineering (CIM 2009), (pp. 236-240).

[14] Grover, S., \& Pea, R. (2013). "Computational thinking in K-12 a review of the state of field." Educational Researcher, 42(1), 38-43. https://doi.org/10.3102/0013189X12463051

[15] Duncan, C., Bell, T., \& Atlas, J. (2017). "What do the teachers think? Introducing computational thinking in the primary school curriculum." ACM International Conference Proceeding Series, 65-74. https://doi.org/10.1145/3013499.3013506

[16] Chalmers, C. (2018). "Robotics and computational thinking in primary school." International Journal of Child-Computer Interaction, 93-100. https://doi.org/10.1016/j.ijcci. 2018.06.005 
[17] Noh, J., \& Lee, J. (2020). "Effects of Robotics Programming on the Computational Thinking and Creativity of Elementary School Students." Association for Educational Communications and Technology 2019.

[18] Umaschi Bers, M., Flannery, L., Kazakoff, E.R., \& Sullivan A. (2014). "Computational Thinking and Tinkering:Exploration of an early childhood robotics curriculum." Computer \& Education, Volume 72, 145-157. https://doi.org/10.1016/j.compedu.2013.10.020

[19] Chen C., Shen J., Barth-CohenbShiyan, L., Jiang, S., Huang, X., \& MoatazEltoukhy, M., (2014). "Assessing Elementary Students' Computational Thinking in Everyday Reasoning and Robotics Programming." Computer \& Education, Volume 109, 162-175. https://doi. org/10.1016/j.compedu.2017.03.001

[20] Joke, V., Fisser, P., Good, J., Mishra, P., \& Yadav, A. (2015). "Computational Thinking in compulsory education:Towards an agenda for research and practice." Education Information Technology, 20(2015), 715-728. https://doi.org/10.1007/s10639-015-9412-6

[21] Bräunl, T. (2006). "Embedded Robotics.” New York: Springer.

[22] Peterson, R. A. (1994). “A Meta-Analysis of Cronbach's Coefficient Alpha.” Journal of Consumer Research, 21(2):381-91. https://doi.org/10.1086/209405

\section{Authors}

Dayang N. A. Jawawi is a Professor in School of Computing, Faculty of Engineering, Universiti Teknologi Malaysia. Her research interests cover software reuse, software quality, software testing, and computing and software engineering education.

Nurul N. Jamal is a postgraduate student in school of computing, Faculty of engineering, Universiti Teknologi Malaysia.

Shahliza Abd Halim is a senior lecturer in School of Computing, Faculty of Engineering, Universiti Teknologi Malaysia. Her research interests cover software reuse, software product line, model driven design and e-learning application.

Nor Azizah Saadon is a senior lecturer in School of Computing, Faculty of Engineering, Universiti Teknologi Malaysia. Her research interests cover web-services, mobile computing, and ontology.

Rosbi bin Mamat is an Associate Professor in School of Electrical Engineering, Faculty of Engineering, Universiti Teknologi Malaysia. His research interests cover control systems, mechatronics, mobile robotics, and software development.

Mohd Adham Isa is a senior lecturer in School of Computing, Faculty of Engineering, Universiti Teknologi Malaysia. His research interests cover software quality, model-driven development, and software development.

Radziah Mohamad is an Associate Professor in School of Computing, Faculty of Engineering, Universiti Teknologi Malaysia. Her research interests cover reusable software, software testing, agent-oriented software engineering, formal methods, and service-oriented computing design.

Haza Nuzly Abdull Hamed is an Associate Professor in School of Computing, Faculty of Engineering, Universiti Teknologi Malaysia. His research interests cover information systems, computational intelligence, optimization, and pattern recognition.

Article submitted 2021-10-05. Resubmitted 2021-11-16. Final acceptance 2021-11-17. Final version published as submitted by the authors. 